\title{
Open Source Vs Proprietary Application and Technologies
}

\author{
Madhu Goel \\ Assistant Professor \\ KITM Kurukshetra
}

\author{
Shobhit Gupta \\ Assistant Professor \\ KITM Kurukshetra
}

\begin{abstract}
In recent decades, every sector of this society has gone computerized. May it be a company, professional organization, institution, hospital, medical store, learning, banking and a large number of crucial sectors, which leads the society to advancement, are almost fully dependent on computers. Every computer or computer-like-device runs on a software system ranging from System software to Software utilities. There are two types of Software providers: Open Source software providers and Proprietary software providers. Majority of society depends on Proprietary category but, by one or the person, people are inspired to move to Open Source by highlighting some of its bigger advantages. But, before shifting entire network or individual system to a new technology, we must study whether the shifting is really worthwhile. In this paper, we will discuss these categories in detail, compare the categories by proposing some ideas and try to make the decision making easier. Light will be thrown on following crucial factors:
\end{abstract}

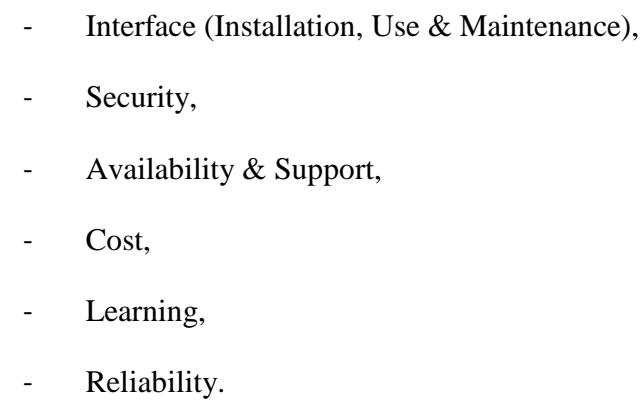

\section{General Terms}

Open Source and Proprietary Software Systems.

\section{Keywords}

Open Source, Proprietary Software, Comparing Open Source $\&$ Proprietary, Types of software, Software Systems, Software

\section{INTRODUCTION}

As already described in the abstract, almost all of the professional and domestic sectors of present society have become computer dependent. By its general definition, as computer is seen as an electronic machine which takes raw data as Input, makes necessary processing on it, save or produces the resultant information in the desired formats. In its initial era, computer was present in the form of several devices like monitor, keyboard, mouse, a central cabinet, separate UPS, etc. But, with the advent of modern computing, the size and shapes of a computer has changed drastically. Now, small computers or computer similar electronic devices are present in our day to day life like Set Top Boxes, Cell Phones, Notebooks, etc. But, being so much different from each other, all these devices still have some major similarities. All of them take input, process information and produce output. All of them interact with humans or other devices to produce desired results. And most important of all, they need Hardware and Software as their major building components.

Hardware is out of the scope of this paper. We will concentrate on Software part here. Software is something which makes a machine turn into computer. It doesn't matter how many devices or how much configuration you have in your machine. No machine of modern era can run without Software. Software is an interface through which a human being interacts with a computer. Without software, working with electronics machine will make computer field equivalent to basic Mechanical or Electronics Engineering. Software is that crucial part of a computer system, which cannot be touched, smelt or felt, but still cannot be ignored. Only the virtual output provided on a screen or physical movements output by some mechanical device can be seen.

Understanding its importance, a large number of crowd is involved in a crucial task of modern computing, The Software Development. Software Development, in itself, is vast subject of research. A lot of software vendors are present in the market that does R \& D work and provides necessary software tools to the society. In general, there are two types of Vendors:

\section{- Vendors who develop Proprietary Software. \\ - Vendors who provide Open Source Software.}

\section{LITERATURE SURVEY}

After collecting information from internet and user experiences, following survey was concluded regarding both types of technologies: 
Table 1: Market Share of Operating Systems

\begin{tabular}{|c|c|c|c|c|c|c|c|c|c|c|c|c|}
\hline \multirow{2}{*}{ Source } & \multirow{2}{*}{ Date } & \multicolumn{4}{|c|}{ Microsoft Windows } & \multicolumn{2}{|c|}{ Apple } & \multicolumn{2}{|c|}{$\stackrel{\text { Linux }}{\text { kernel based }}$} & \multirow{2}{*}{ Symbian } & \multirow{2}{*}{$\begin{array}{l}\text { Black- } \\
\text { Berny } \\
\text { os }\end{array}$} & \multirow{2}{*}{ Other } \\
\hline & & $\underline{7}$ & Vista & $\underline{X P}$ & $\begin{array}{c}\text { All } \\
\text { versions }\end{array}$ & os $x$ & $\underline{i o s}$ & $\frac{\text { GNU }}{\text { Linux }}$ & Android & & & \\
\hline AT Internet ${ }^{113]}$ & Aug. 2011 & $33.0 \%$ & $14.7 \%$ & $38.4 \%$ & $87.1 \%$ & $\square 6.7 \%$ & $3.7 \%$ & $1.0 \%$ & $0.9 \%$ & - & - & $0.6 \%$ \\
\hline Clicky Web Analytics $[141$ & Oct. 2011 & $33.67 \%$ & $10.17 \%$ & $26.11 \%$ & $79.53 \%$ & $13.25 \%$ & $3.81 \%$ & $1.16 \%$ & $1.77 \%$ & $0.12 \%$ & $0.32 \%$ & $0.04 \%$ \\
\hline Chitika[15] & Oct 2011 & - & - & - & $77.2 \%$ & $10.2 \%$ & $5.4 \%$ & $2.9 \%$ & $3.3 \%$ & - & - & $1.0 \%$ \\
\hline Net Market Share[16][17] & Feb. 2012 & $35.25 \%$ & $\square 7.49 \%$ & $41.98 \%$ & $85.01 \%$ & $\square 6.4 \%$ & $4.38 \%$ & $1.07 \%$ & $1.38 \%$ & $0.2 \%$ & $0.16 \%$ & $1.4 \%$ \\
\hline StatCounter Global Stats[18I119120] & Feb. 2012 & $40.93 \%$ & $8.84 \%$ & $30.76 \%$ & $82.11 \%$ & 口6.78\% & $3.83 \%$ & $0.71 \%$ & $2.11 \%$ & $2.58 \%$ & $0.58 \%$ & $1.30 \%$ \\
\hline StatOw|[21] & Oct. 2011 & $33.24 \%$ & $12.91 \%$ & $33.20 \%$ & $78.94 \%$ & $14.04 \%$ & - & $0.70 \%$ & - & - & - & $6.32 \%$ \\
\hline W3Counter[22] & Oct. 2011 & $36.36 \%$ & $\square 9.57 \%$ & $34.18 \%$ & $80.21 \%$ & 口8.86\% & $3.21 \%$ & $1.65 \%$ & $1.29 \%$ & $0.16 \%$ & $0.60 \%$ & $4.02 \%$ \\
\hline Webmasterpro[31 & Nov. 2011 & $38.3 \%$ & $14.1 \%$ & $32.1 \%$ & $85.8 \%$ & 口6.3\% & $4.4 \%$ & $1.4 \%$ & $1.6 \%$ & $0.2 \%$ & $0.02 \%$ & $0.28 \%$ \\
\hline Wikimedia[24] & Mar. 2012 & $37.13 \%$ & 口8.31\% & $26.78 \%$ & $73.38 \%$ & 口8.41\% & $7.46 \%$ & $1.59 \%$ & $3.46 \%$ & $0.16 \%$ & $0.81 \%$ & $4.73 \%$ \\
\hline
\end{tabular}

\subsection{Proprietary Software}

The term proprietary is derived from the Latin word proprietas meaning property [2]. Proprietary Software is computer software licensed under the exclusive legal rights of the copyright holder. Proprietary software is developed by a person or firm who has rights of using existing or developing new tools to create new software. A proprietary software developer sells or provides his creation under some concrete conditions which should be followed in order to avoid any legal issues. In general, these concrete conditions involves usage using software with a purchased license, within the permitted boundaries, no modification allowed, no further redistributions and no reverse engineering to applied[1]. The main identity of proprietary software is that its source code is always kept secret form outside world. Thus, the internal structure of proprietary software is not exposed. The restrictions on proprietary software are generally imposed through a document called EULA (End-user license agreements) to which users are supposed to agree before using the software [2]. It works just like a contract of usage conditions between the user and vendor. If a user is found indulged in activities leading to the breaking of copyright conditions, the selling authority has the right to impose legal actions against the misusing personal.

Based on availability and usability, various kinds of proprietary software are:

Freeware: It is provided to be used without any monetary charges. However, severe restrictions of its use, modification and redistribution are still imposed and Source code is not provided. It can be passed on to anyone free of cost. [2]
Shareware: It is generally that version of software which is limited in terms of functionality. Users can download it from internet, uses it with restrictions and then decide to pay for its full version. These can be shared among other people.

Trialware: It is generally that version of software which is limited in terms of duration of use. User gets the full version of software which can be limited to the usage number of days or usage number of times. After paying and getting registered, the user will get unrestricted access to usage and updates of that software.

- Public Domain Software: The copyright holder donates it to the public. Thus, it is no longer copyrighted and restricted and thus moves towards Open source.[2]

\subsection{Open Source Software}

As the name suggests, source code of such software is exposed. Open source is a methodology or approach towards the design and development of software with the intention of giving the user access to source code. These type of software exposes their inter structures such that the user can use it, debug it, modify it and even redistribute it with his own modifications. [3] Open source software developed, tested and distributed in an open environment without the label of any particular company which can be used for marketing purposes. Open source software are used and distributed under various types licenses stated below: 
- GNU General Public License (GPL): Also called the 'Copyleft' license, states that if a user wants to modify and launch his own version of a software, it should be done under the same GNU-GPL license thus, keeping the resulting software also free of cost.

- GNU Lesser GPL: The copyright notices and disclaimer must be preserved and further use of source code is unrestricted for both proprietary \& open source programmes.

BSD license: Completely unrestricting license allow the use of code or its modification in any possible way.

- Market share of Proprietary \& Open Source Software.

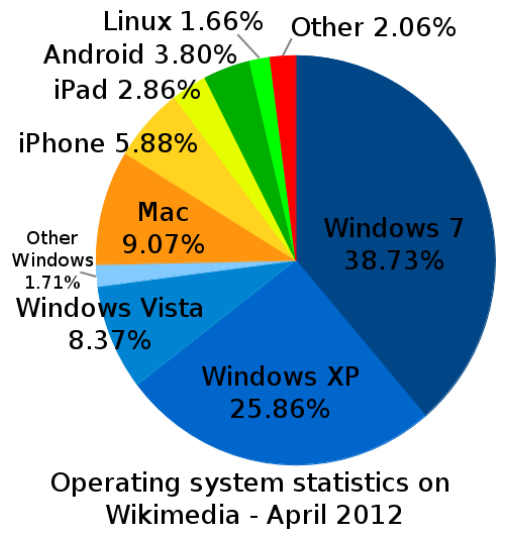

Figure 1: Usage share of Operating Systems:

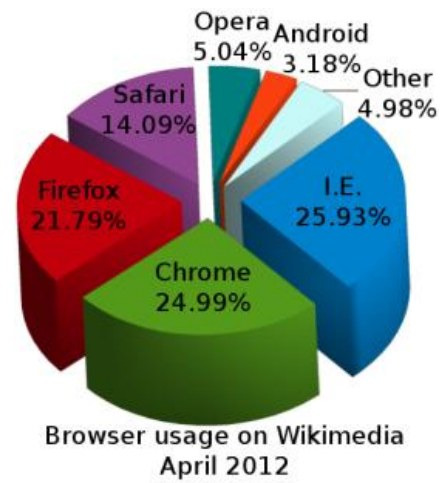

Figure 2: Usage Share of Browsers
Table 2: Usage share of desktop browsers for March 2012

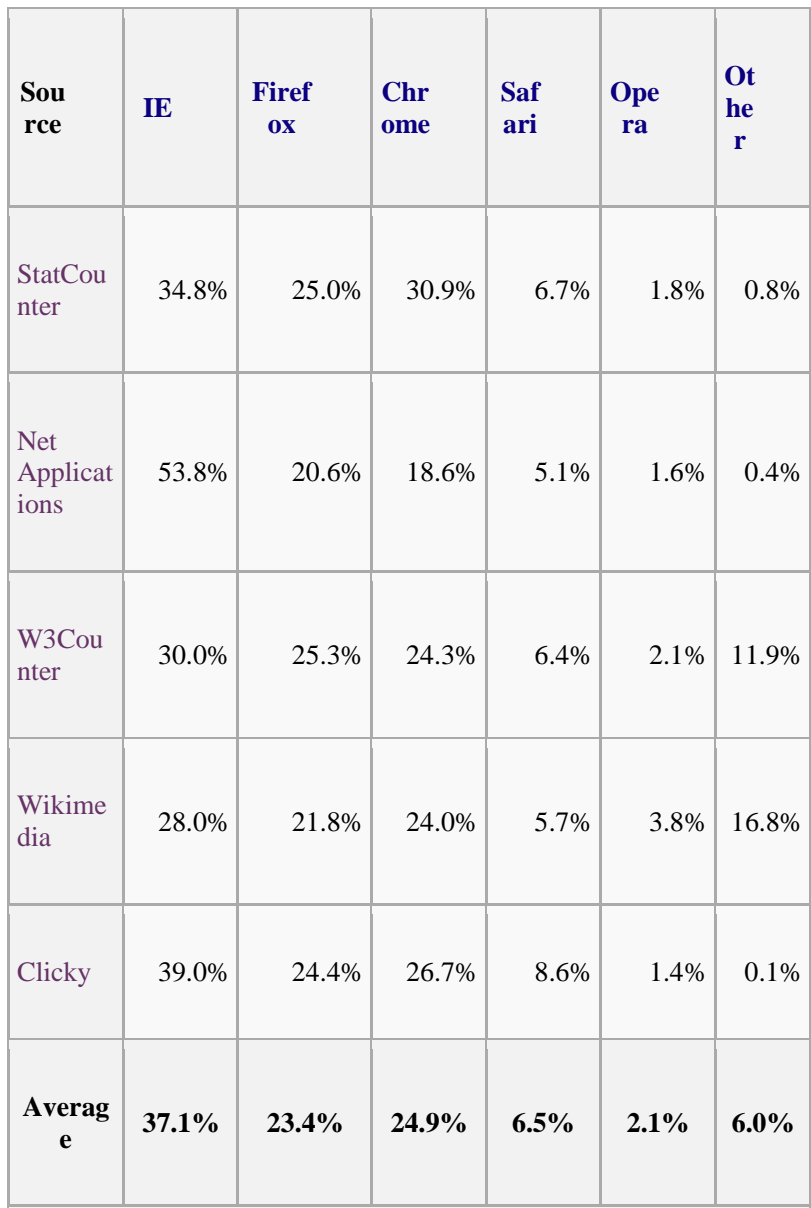

Table 3: Usage share of desktop browsers for March 2012

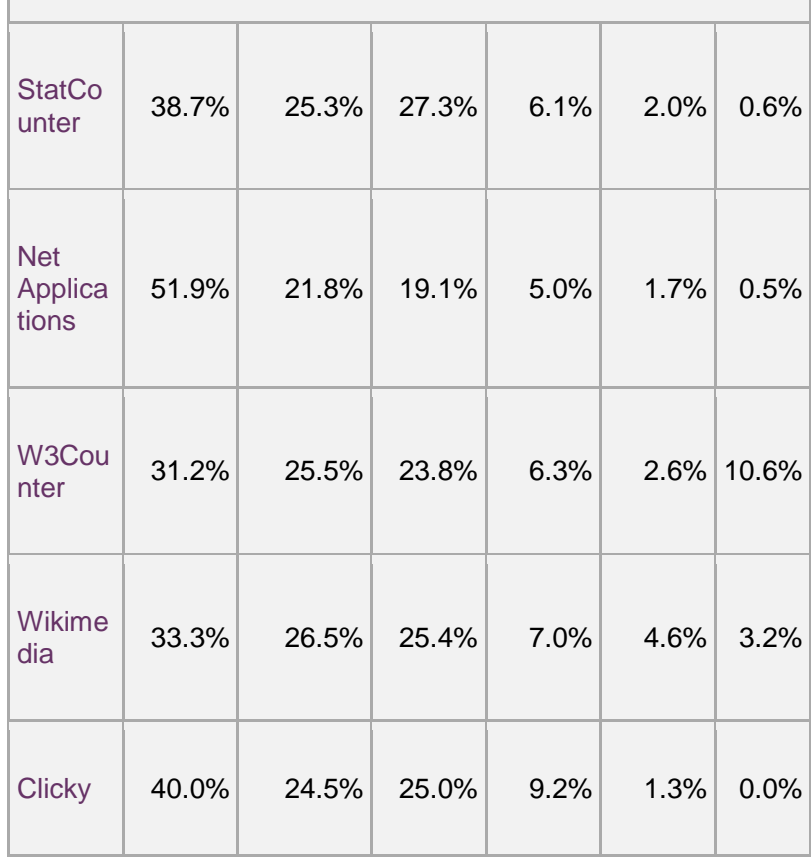




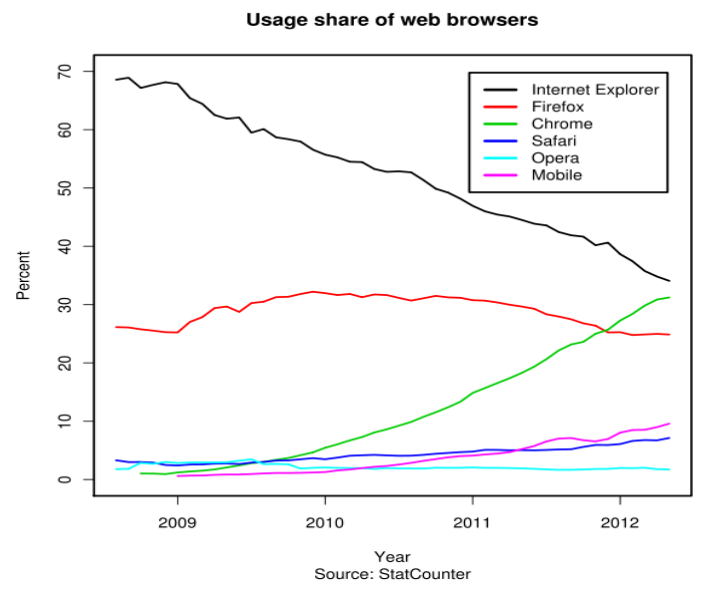

Figure 3: Comparative Usage Share of Web Browsers

\section{PROPOSED DISCUSSION}

Before choosing any technology domain, we should know how the technology performs among various critical factors. In this paper, we try to elaborate the discussion which will help in making the right choice. In this discussion we will consider the leading brands: LINUX \& Microsoft WINDOWS as main competitors.

\subsection{Availability:}

The proprietary softwares (PS) are available at most computer accessories outlet. Any consumer can visit a shop dealing with computer accessories and purchase a license and CD of the product. Such softwares are available as packages according to different levels of consumer needs with reasonable rates. On the other hand, Open Source Softwares (OSS)are freely available over internet and may be downloaded and used without any license restrictions. Both have different advantages under this factor. Free availability of OSS provides flexibility to consumer whereas, PS provides reliability. The packed product provides satisfaction while, download links may be fake or hacked. But, some OSS products provide a different medium of availability. For example, user can fill a form and order free copy of Linux (Ubuntu) at residence without any postage charges. But, not all products are available in a similar way.

\subsection{Transparency:}

The source code of OSS is freely available along with the product. Any person can read, modify, build and distribute a modified version of original product. Thus, it gives a transparent look at the core structure of the product. On the other hand, PS does not provide an open look to the internal structure. Only user interfaces are provided to work with it. User cannot know the internal processing and other details.

\subsection{Reliability:}

In connection with the previous factors, since OSS are available on a large number of unverified websites and even most of these distributions may be modified by any technologically sound user, all the distributions are reliable in terms of security, robustness, performance. The reason is that is a user adds/modifies some component; it may works good individually, but, may clash with other components and ultimately degrade the product. At contrast, a PS is developed by specialized teams at vendor's end only. Only finished products are provided at outlets. Since there is no unauthenticated modification, the outcome is always reliable.

\subsection{Security:}

This factors works in collaboration with previous ones. OSS is supposed to be secure, for example LINUX. But, a crucial argument is always ignored in this factor. LINUX operating system, considered to be the best OS product, is widely used on server end. Server systems are very critical and delicate. Each and every information bit needs to be highly secured. Thus, companies handling Server machines, rely on branded OS products only. Downloaded or openly modified OS products are not used. Thus, picture becomes similar to PS systems where the product is purchased from branded company only, for example Windows from Microsoft in this case. So, truly OS systems do not some into real picture. Also, products purchased from branded company will not be hacked easily. Vulnerable systems are those downloaded from unauthenticated sources. Original OSS is secured by company directly. Also, since PS is not widely used by normal computers, either security breaches are not detected, or they are not present. This is because, server administration organization purchases original product, which do not have loopholes. General purpose users do not work on LINUX much due to less friendly environment or lack of knowledge. That's why, neither much viruses are created, nor hackers bother about it since the probability of getting hacked information is very less due to less number of users. So, when OSS products will increase in use, security threat will increase wit high rates.

\subsection{User-friendly:}

OSS is less user friendly as compared to PS products. PS provides more user interface components in an easy-to-use fashion. Again, this factor depends upon discussion made in security. PS like Windows, is widely used by end-users thus, more stress is laid on interface rather than processing power. On the other hand, OSS is used mainly on servers where userinterface attractiveness doesn't matter. Computer power is the most crucial factor on server end. Even PS made for server systems like Windows 2005, 2008 Server Editions do not have an attractive interface.

\subsection{Maintenance \& Support:}

In PS, there is brand vendor who provides a product. So, it is always easy to find them for getting support. Also, the purchasing cost of PS products includes maintenance/support charges. While, in case of OSS, the products are downloaded from internet which were developed by a community of developers who are not employees. It is hard to get the problems solved from open developers. Although there are companies who promise to provide support for the product, but they impart heavy charges for the same. While, PS vendors provide support almost free of cost since it was promised during the purchase.

\subsection{Cost:}

The PS are paid products. Consumer need to pay and purchase the product for use. On the other hand, an OSS are available free of cost. User can directly download and use the product with limited or no conditions depending upon the distribution licensing. But, if user needs some help and support, he needs to pay for it. Thus, OSS are not purely free since, maintenance $\&$ support charges are required to be paid which makes it expensive than PS. 


\section{CONCLUSION}

PS and OSS have advantages \& disadvantages depending upon the application features they provide. PS have a long lasting stand in the computing era. They are also very secure and user-friendly. These systems have been serving a wide range of society for decades. OSS, on the other hand, is a new and emerging idea for many decades. Regular user markets have not been convinced by the ideas of OSS systems. From a general user point of view, a PS product is found still more reliable and effective as compared to the OSS. On contrast, OSS products are far more reliable and effective for critical networking and other applications due to their robustness and efficient performance. OSS still has a long way to go in order to become a regular demand of a daily user. After taking this discussion into account, user may get an idea about what type of software system should be selected for what purpose and OSS developers may get some idea about the improvements required to make a strong hold in the software market in future.

\section{ACKNOWLEDGMENTS}

We pay regards and thanks to our parents who made us stand on our feet and supported us through all the phase of our lives. We are also thankful to the Director-Principal of KITM for his moral support. We are thankful to our family and all the supporters. We also pay thanks to God for making us able to fulfill the expectations of related ones.

\section{REFERENCES}

[1] Digit Fast Track to open Source Software Vol. 4 Issue 8.

[2] http://en.wikipedia.org/wiki/Proprietary_software

[3] http://www.linfo.org/proprietary.html

[4] http://opensource.org/

[5] http://www.webopedia.com/TERM/O/open_source.html

[6] http://c2.com/cgi/wiki?ProprietarySource

[7] http://ostatic.com/question/open-source-v-s-proprietarysource-advantages-disadvantages

[8] http://www.getclicky.com/marketshare/global/webbrowsers/

[9] http://en.wikipedia.org/wiki/Usage_share_of_web_brows ers 